\title{
The New Era of Multiple Sclerosis Therapeutics
}

\author{
Rohit Bakshi
}

Published online: 13 December 2012

(C) The American Society for Experimental NeuroTherapeutics, Inc. 2012

The early 1990s began a new era in the treatment of MS, when the FDA approved interferon beta- $1 b$ as the first treatment for reducing the rate of relapses. Many additional effective therapies have followed during the past 20 years, each of which was shown in clinical trials to reduce both the clinical and neuroimaging abnormalities associated with disease progression. These medications clearly limited the impact of the disease as shown by placebo-controlled trials, reducing the relapse rate by up to two-thirds, and the risk of sustained progression of disability by up to $40 \%$. The available therapies offer a wide array of options, with diverse mechanisms of action and a range of treatment routes and frequency of administrations. In this volume, we present the current state and future prospects of MS therapies.

We include 12 critical reviews in this volume, written by leading authorities. The volume begins with assessment of currently available immunomodulatory agents, followed by a review of pipeline therapies, such as those currently in phase II/III trials or filed with the FDA. A number of subsequent papers focus on the role of aggressive immunosuppressive and stem-cell strategies, or use of immunotherapies in children with MS. These are followed by reviews of symptomatic and acute relapse treatments. The volume concludes with a review of cutting-edge imaging strategies to non-invasively monitor MS-related neuroinflammation. Although my wish as Guest Editor was to make this edition comprehensive, we were not able to cover all of the available and emerging therapies due to publication deadlines.

Despite the abundant progress made in this new MS neurotherapeutic era, there remain several unmet needs. For early stage relapsing forms of MS, the treatment effects are significant but only partial. For progressive forms of MS, these agents are generally ineffective with regard to limiting disease progression. When considering the use of such therapies, patients and caregivers must weigh benefits against treatment burdens such as route/ frequency of administration, side effects, and safety. Thus, there remains a "treatment gap" that provides a challenge to investigators in the field. Nonetheless, the availability of current therapies has made MS a treatable, manageable disease.

\section{Rohit Bakshi}

Guest Editor

\footnotetext{
R. Bakshi $(\bowtie)$

Department of Neurology, Brigham and Women's Hospital, Partners MS Center, Harvard Medical School,

One Brookline Place,

Brookline, MA 02445, USA

e-mail: rbakshi@bwh.harvard.edu
} 